\title{
Campagne d'échantillonnage et de mesure de champignons et de baies dans un massif forestier du Parc National du Mercantour
}

\author{
Ph. CALMON*
}

(Manuscrit reçu le 16 septembre 1998, révisé le 27 mai 1999, accepté le 3 septembre 1999)

RÉSUMÉ Lors du passage du nuage de Tchernobyl, en France, les dépôts ont été les plus élevés dans la partie Est du pays. Dû à des précipitations importantes entre le $1^{\mathrm{er}}$ et le 5 mai 1986, notamment dans le Mercantour, cette région a reçu des dépôts significatifs en césium. En septembre 1997, en collaboration avec le Parc National du Mercantour, une campagne d'échantillonnage et de mesure de champignons et de baies a été mencé dans la forêt du Boréon, entre 1500 et $1700 \mathrm{~m}$ d'altitude. La concentration maximum en ${ }^{137} \mathrm{Cs}$ dans des champignons a été : $130,3 \pm 5,5 \mathrm{~Bq} \mathrm{~kg}^{-1}$ frais pour des Lactaires délicieux et la concentration minimum dans des Coulemelles : $1,0 \pm 0,1 \mathrm{~Bq} \mathrm{~kg}^{-1}$ frais et dans les baies : $1,8 \pm 0,1 \mathrm{~Bq} \mathrm{~kg}^{-1}$ frais pour des Myrtilles. Dans les sols, les activités surfaciques déterminées pour le même radionucléide ont

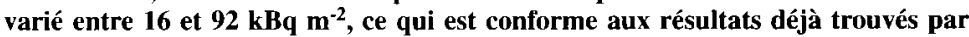
l'IPSN dans ce secteur. La part des retombées anciennes par rapport à Tchernobyl est en moyenne de $40 \%$ des valeurs totales mesurées en ${ }^{137} \mathrm{Cs}$.

ABSTRACT Sampling and measuring operation for mushrooms and berries in a forested massif belonging to the National Parc of Mercantour.

When the Chernobyl plume crossed France, most of the depasition acted in the eastern part of the country. Because of heavy precipitations, between the first and the fifth of May 1986, especially in the Mercantour, this region received significant deposition levels of cesium. In September 1997, in collaboration with the National Parc of Mercantour, a sampling and measuring operation for mushrooms and berries was conducted in the Boréon forest, between 1500 and 1700 meters high. The maximal concentration of ${ }^{137} \mathrm{Cs}$ in mushrooms was: $130.3 \pm 5.5 \mathrm{~Bq} \mathrm{~kg}^{-1}$ fresh weight, for Lactarius deliciosus and the minimum concentration in Macrolepiota procera: $1.0 \pm 0.1 \mathrm{~Bq} \mathrm{~kg}^{-1}$ fresh weight and in berries: $1.8 \pm 0.1 \mathrm{~Bq} \mathrm{~kg}^{-1}$ fresh weight for Vaccinium myrtillus. In soils, the surfacic concentrations found for the same radionuclide varied between 16 and $92 \mathrm{kBq} \mathrm{m}^{-2}$. This is in accordance with other results already identified by IPSN, in this site. On the average, $40 \%$ of the measured concentrations of ${ }^{137} \mathrm{Cs}$ can be attributed to the nuclear weapons test fallout and the rest to the Chernobyl fallout.

\footnotetext{
* IPSN, Institut de protection et de sûreté nucléaire, département de protection de l'environnement, service d'études et de recherches radioécologiques de laboratoire, C.E. Cadarache, 13108 Saint-Paul-lez-Durance Cedex. France.
} 


\section{Matériel et méthodes}

Une campagne d'échantillonnage a été organisée le 3 septembre 1997 en collaboration avec le Parc National du Mercantour. Les prélèvements ont été effectués dans le Massif du Boréon, au-dessus de St Martin-Vésubie, depuis la vallée du Boréon jusqu'au vallon de Salèse.

Cinq espèces de champignons (bolets, coulemelles, chanterelles, girolles et lactaires) et une espèce de baies (myrtilles) ont été collectées. À chaque fois que cela a été possible, le sol sous-jacent ou proche a été prélevé en même temps, selon des couches successives de $5 \mathrm{~cm}$ jusqu'à une profondeur maximale de $25 \mathrm{~cm}$. Pour ce faire, un carottier (tube inox de $7,3 \mathrm{~cm}$ de diamètre) a été utilisé.

Les échantillons ramenés au laboratoire ont été pesés immédiatement afin de déterminer le poids frais. Les champignons et les baies ont été lavés à l'eau douce pour les débarrasser de particules de terre, de débris végétaux ou de feuilles. Les couches de sol ont subi un tamisage à $2 \mathrm{~mm}$ afin de retirer les cailloux et divers débris végétaux. Tous les échantillons ont ensuite été séchés à l'étuve à $105^{\circ} \mathrm{C}$ jusqu'à obtention d'un poids constant. Une fois séchés, tous les échantillons ont été pesés afin de déterminer le poids sec.

Les échantillons ont ensuite été broyés, à l'aide d'un broyeur à couteaux, afin d'obtenir une poudre fine et homogène. Cette poudre a été répartie et tassée dans des géométries de $20,60,200$ et $380 \mathrm{ml}$, selon les quantités disponibles pour chaque échantillon. Les mesures d'activité ont été effectuées par le laboratoire de mesure de la radioactivité de l'environnement, appartenant à l'IPSN et situé à Orsay (91).

Les échantillons ont été analysés par spectrométrie- $\gamma$ Germanium bas niveau, avec un temps de comptage de 8000 secondes.

\section{Résultats}

\subsection{Concentrations en ${ }^{40} \mathrm{~K},{ }^{134} \mathrm{Cs}$ et ${ }^{137} \mathrm{Cs}$ des champignons et des baies}

Dans le tableau I, sont rassemblés les résultats des mesures en ${ }^{40} \mathrm{~K}$, radionucléide naturel le plus représenté dans la biosphère, et en ${ }^{134} \mathrm{Cs}$ et ${ }^{137} \mathrm{Cs}$, radionucléides artificiels majoritairement déposés après l'accident de Tchernobyl. Afin de concentrer les radionucléides et d'abaisser le seuil de détection, tous les échantillons ont été mesurés en sec. Connaissant les rapports poids frais/poids sec de chacun des échantillons, les concentrations en ${ }^{137} \mathrm{Cs}$ ont pu être calculées en référence au poids frais. 


\section{TABLEAU I}

Concentrations en ${ }^{40} \mathrm{~K},{ }^{134} \mathrm{Cs}$ et ${ }^{1337} \mathrm{Cs}$ de quelques espèces de champignons et de baies, récoltés dans le Massif du Boréon.

Concentrations in ${ }^{40} \mathrm{~K},{ }^{134} \mathrm{Cs}$ and ${ }^{137} \mathrm{Cs}$ of some species of mushrooms and berries collected in the Boreon massif.

\begin{tabular}{|c|c|c|c|}
\hline Espèces & $\begin{array}{l}\text { Concentration } \\
\mathrm{en}^{40} \mathrm{~K} \\
\left(\mathrm{~Bq} \mathrm{~kg}{ }^{-1} \text { frais }\right)\end{array}$ & $\begin{array}{l}\text { Concentration } \\
\text { en }{ }^{134} \mathrm{Cs} \\
\left(\mathrm{Bq} \mathrm{kg}^{-1} \text { frais) }\right.\end{array}$ & $\begin{array}{c}\text { Concentration } \\
\text { en }{ }^{137} \mathrm{Cs} \\
\left(\mathrm{Bg} \mathrm{kg}^{-1} \text { frais) }\right.\end{array}$ \\
\hline Cèpes n¹ (Boletus edulis) & $86,4 \pm 5,8$ & $<0.23$ & $14,4 \pm 0,8$ \\
\hline Coulemelles (Macrolepiota procera) & $111,6 \pm 6,2$ & $<0,12$ & $1,0 \pm 0,1$ \\
\hline Cèpes n² (Boletus edulis) & $85,4 \pm 4,9$ & $0,22 \pm 0,06$ & $32,7 \pm 1,5$ \\
\hline Cèpes n̊3 (Boletus edulis) & $75,6 \pm 4,4$ & $0,28 \pm 0,04$ & $34,2 \pm 1,4$ \\
\hline Cèpes n4 (Boletus edulis) & $63,2 \pm 4,0$ & $0,22 \pm 0,04$ & $46,6 \pm 2,0$ \\
\hline Chanterelles (Cantharellus lutescens) & $55,6 \pm 3,3$ & $0,84 \pm 0,06$ & $106,7 \pm 4,4$ \\
\hline Girolles $\mathrm{n}^{\circ} 1$ (Cantharellus cibarius) & $91,8 \pm 6,2$ & $0,29 \pm 0,03$ & $54,6 \pm 3,1$ \\
\hline Girolles $\mathrm{n}^{\circ} 2$ (Cantharellus cibarius) & $92,4 \pm 5,4$ & $0,67 \pm 0,07$ & $67,2 \pm 3,0$ \\
\hline Lactaires délicieux (Lactarius deliciosus) & $70,3 \pm 4,7$ & $1,64 \pm 0,12$ & $130,4 \pm 5,5$ \\
\hline Myrtilles (Vaccinium myrtillus) & $23,4 \pm 1,7$ & $<0,05$ & $1,8 \pm 0,1$ \\
\hline
\end{tabular}

Ces résultats montrent que les concentrations en ${ }^{40} \mathrm{~K}$ ne présentent qu'une variation faible d'environ un facteur 2,2 entre les différentes espèces de champignons. La concentration en ${ }^{40} \mathrm{~K}$ des myrtilles est ici 4 à 5 fois plus faible que la moyenne de celle des champignons, ce qui est dû à la composition naturelle en éléments essentiels propre à chaque type de végétal. Les concentrations en ${ }^{137} \mathrm{Cs}$ des différentes espèces de champignons sont nettement plus variables, d'un facteur 130, comparativement à celles du ${ }^{40} \mathrm{~K}$.

Les niveaux de concentration en ${ }^{137} \mathrm{Cs}$ des cèpes, des chanterelles, des girolles et des lactaires sont détectables et présentent des niveaux significatifs, cependant au minimum 10 fois inférieurs à la limite de $1,250 \mathrm{~Bq} \mathrm{~kg}^{-1}$ frais, fixée le 22/07/1989 par la Directive CEE pour les produits alimentaires autres que les produits laitiers, les boissons et les aliments pour nouveau-nés et relatifs aux isotopes du césium.

\subsection{Concentrations en ${ }^{40} \mathrm{~K},{ }^{134} \mathrm{Cs}$ et ${ }^{137} \mathrm{Cs}$ des sols}

Dans la mesure du possible, des échantillons de sol ont systématiquement été pris sous, ou à proximité, des points de collecte des champignons et des myrtilles. Les sols ont été prélevés à l'aide d'un carottier. La présence d'une trop grande quantité de pierres a empêché le prélèvement du sol pour les cèpes $\mathrm{n}^{\circ} 2$ et a contraint à ne prélever que la première couche de sol pour les cèpes $n^{\circ} 4$, les chanterelles et les girolles $n^{\circ} 1$. Les prélèvements de sol se sont déroulés par couches successives de $5 \mathrm{~cm}$, jusqu'à $25 \mathrm{~cm}$ de profondeur, lorsque le terrain l'a permis. 


\section{Concentration en cesium-137 \\ (Bq kg-1sec)}

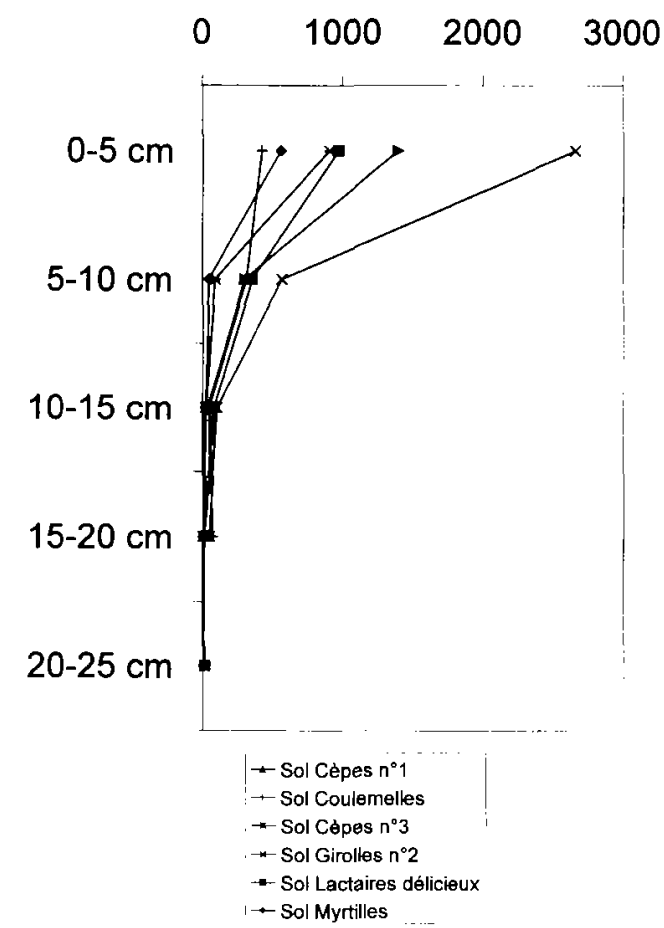

Fig. I. - Concentrations en ${ }^{137}$ Cs de différents horizons successifs de sol dans le massif du Boréon.

${ }^{137} \mathrm{Cs}$ concentrations in several successive layers of soil in the Boreon massif.

Les concentrations en ${ }^{40} \mathrm{~K}$ sont homogènes entre les différents sols et ne varient pas en fonction de la profondeur de sol. Elles se situent en moyenne à $889 \pm$ $58 \mathrm{~Bq} \mathrm{~kg}^{-1} \mathrm{sec}$. En revanche, les concentrations en ${ }^{137} \mathrm{Cs}$ diminuent fortement avec la profondeur, comme l'illustre la figure 1.

Connaissant le poids de chaque couche de sol prélevée ainsi que le volume défini par la section du carottier, il est possible de rapporter la concentration en ${ }^{137} \mathrm{Cs}$ à une surface de $1 \mathrm{~m}^{2}$. Les résultats sont présentés dans le tableau II.

\section{Discussion}

\subsection{Rapports isotopiques des profils de sols}

Lorsque les concentrations en ${ }^{134} \mathrm{Cs}$ sont détectables, il est possible de calculer les rapports isotopiques ${ }^{137} \mathrm{Cs} /{ }^{134} \mathrm{Cs}$ afin de déterminer l'origine du césium. Lors de 


\section{TABLEAU II}

Contamination surfacique en ${ }^{137} \mathrm{Cs}$ des couches de sols sur lesquels ont été récoltés les champignons et les baies, en septembre 1997, dans le massif du Boréon.

Surfacic contamination in ${ }^{137} \mathrm{Cs}$ of soil layers where mushrooms and berries were collected in September 1997, in the Boreon massif.

\begin{tabular}{|c|c|c|}
\hline Sols & 'rofondeur de sol prélevé & $\begin{array}{l}\text { Contamination surfacique } \\
\qquad \text { en }{ }^{137} \mathrm{Cs}\left(\mathrm{Bq} \mathrm{m} \mathrm{m}^{-2}\right)\end{array}$ \\
\hline Sol Cèpes n ${ }^{\circ} 1$ & $0-15 \mathrm{~cm}$ & 27,416 \\
\hline Sol Coulemelles & $0-20 \mathrm{~cm}$ & 24,474 \\
\hline Sol Cèpes n ${ }^{\circ}$ & - & ND \\
\hline Sol Cèpes n ${ }^{\circ} 3$ & $0-25 \mathrm{~cm}$ & 15,671 \\
\hline Sol Cèpes $n^{\circ} 4$, Chanterelles et Girolles $n^{\circ}$ & $0-10 \mathrm{~cm}$ & 29,597 \\
\hline Sol Girolles $\mathrm{N}^{\circ} 2$ & $0-20 \mathrm{~cm}$ & 80,702 \\
\hline Sol Lactaires délicieux & $0-25 \mathrm{~cm}$ & 91,925 \\
\hline Sol Myrtilles & $0-25 \mathrm{~cm}$ & 31,924 \\
\hline
\end{tabular}

l'accident de Tchernobyl, deux isotopes radioactifs du césium de périodes longues ont été rejetés dans l'atmosphère : $1 e^{134} \mathrm{Cs}$ (Période $=2,06$ ans) et le ${ }^{137} \mathrm{Cs}$ ( 30 ans). Début mai 1986, si la mesure d'un échantillon quelconque issu de l'environnement montrait entre les deux isotopes un rapport de concentration égal à 2,1 , l'origine d'une contamination due à Tchernobyl était indiscutable. Le ${ }^{134} \mathrm{Cs}$ décroît plus rapidement au cours du temps que le ${ }^{137} \mathrm{Cs}$, ainsi le rapport spécifique tchernobylien augmente régulièrement avec le temps et a atteint la valeur de 72,1 début septembre 1997, date à laquelle ont été effectués les prélèvements.

La contribution de l'accident de Tchernobyl dans les sols évolue de 37 à $85 \%$, mais tous sols et couches de sol confondus, elle ne dépasse pas $60 \%$, en moyenne. Ce qui signifie que les $40 \%$ restant proviennent des retombées des anciens tirs d'essais d'armes atomiques effectués dans les années 1960.

Les rapports isotopiques dans les sols augmentent en fonction de la profondeur, indiquant ainsi que le césium provenant de l'accident de Tchernobyl n'a pas migré aussi profondément que celui provenant des anciens tirs.

Si l'on compare les rapports isotopiques des champignons avec ceux des sols (Tab. III), il est possible d'estimer la profondeur de la couche de sol où se situent le mycélium du champignon. Malheureusement, des concentrations en ${ }^{134} \mathrm{Cs}$ n'ont pas pu être détectées dans chaque couche de sol. Les lactaires délicieux, ayant un rapport isotopique très proche du premier horizon de sol, ont très probablement leur mycélium très superficiel. En revanche, les girolles semblent avoir un mycélium beaucoup plus profond, en dessous des 10 premiers centimètres du sol. 
TABLEAU III

Rapports isotopiques du césium de quelques champignons et de sols prélevés dans le massif du Boréon, en septembre 1997.

Cesium isotopic ratios for some mushrooms and soils sampled in the Boreon massif, in September 1997.

\begin{tabular}{|c|c|c|c|}
\hline & & \multicolumn{2}{|c|}{ Rapports isotopiques ${ }^{137} \mathrm{Cs} /{ }^{134} \mathrm{Cs}$} \\
\hline & & Champignons et baies & Sols \\
\hline Girolles $\mathbf{n}^{\circ} 2$ & & 100 & \\
\hline \multirow[t]{4}{*}{ Sol Girolles n² : } & $0-5 \mathrm{~cm}$ & & 85 \\
\hline & $5-10 \mathrm{~cm}$ & & 93 \\
\hline & $10-15 \mathrm{~cm}$ & & 129 \\
\hline & $15-20 \mathrm{~cm}$ & & 114 \\
\hline Lactaires délicieux & & 80 & \\
\hline \multirow[t]{5}{*}{ Sol Lactaires délicieux : } & $0-5 \mathrm{~cm}$ & & 85 \\
\hline & $5-10 \mathrm{~cm}$ & & 104 \\
\hline & $10-15 \mathrm{~cm}$ & & 104 \\
\hline & $15-20 \mathrm{~cm}$ & & ND \\
\hline & $20-25 \mathrm{~cm}$ & & ND \\
\hline
\end{tabular}

ND: non déterminé, car pas de concentration détectable en ${ }^{134} \mathrm{Cs}$.

\subsection{Facteurs et coefficients de transfert aux champignons et aux baies}

Afin de rendre compte des transferts entre les sols et les champignons ou les baies, on peut utiliser soit le facteur de transfert, soit le coefficient de transfert selon que l'on rapporte la contamination du sol soit par rapport à la masse de terre, soit par rapport à une surface d'un $\mathrm{m}^{2}$.

Le facteur de transfert est le rapport entre la concentration du radionucléide dans les champignons ou les baies et celle dans le sol. L'unité est le $\mathrm{Bq} \mathrm{kg}-1 / \mathrm{Bq} \mathrm{kg}^{-1}$.

Le coefficient de transfert est le rapport entre la concentration du radionucléide dans les champignons ou les baies et celle rapportée à une surface de $1 \mathrm{~m}^{2}$ de sol. L'unité est le $\mathrm{m}^{2} \mathrm{~kg}^{-1}$.

L'AIEA (AIEA, 1994) recommande l'utilisation d'un coefficient de transfert global, exprimé en $\mathrm{m}^{2} \mathrm{~kg}^{-1}\left(\mathrm{~Bq} \mathrm{~kg}^{-1} / \mathrm{Bq} \mathrm{m}^{-2}\right)$ car, précisent Fawaris et Johanson (1995), dans les études sur le terrain, l'utilisation du coefficient de transfert ( $\mathrm{en} \mathrm{m}^{2} \mathrm{~kg}^{-1}$ ) est préférable, principalement à cause de la répartition hétérogène du radiocésium en forêt et le manque de connaissance de la répartition en profondeur du mycélium des champignons impliqués dans l'absorption du radiocésium. 


\section{TABLEAU IV}

Facteurs de transfert du ${ }^{137} \mathrm{Cs}$ et $\mathrm{du}{ }^{40} \mathrm{~K}$ et coefficient de transfert du ${ }^{137} \mathrm{Cs}$ de champignons et de baies prélevés dans le massif du Boréon, en septembre 1997.

Transfer factors for ${ }^{137} \mathrm{Cs},{ }^{40} \mathrm{~K}$ and transfer coefficients of ${ }^{137} \mathrm{Cs}$ for mushrooms and berries sampled in the Boreon massif, in september 1997.

\begin{tabular}{|c|c|c|c|c|}
\hline \multicolumn{2}{|l|}{ Sols } & $\begin{array}{l}\text { Facteur de } \\
\text { transfert } \mathrm{du}^{40} \mathrm{~K} \\
(\mathrm{~Bq} \mathrm{kg-1} \text { frais } \\
\left./ \mathrm{Bq} \mathrm{kg}^{-1} \text { sol sec) }\right)\end{array}$ & $\begin{array}{l}\text { Facteur de transfert } \\
\qquad \mathrm{du}^{137} \mathrm{Cs} \\
\left(\mathrm{Bq} \mathrm{kg} \mathrm{kg}^{-1} \text { frais }\right. \\
/ \mathrm{Bq} \mathrm{kg}^{-1} \text { sol sec) }\end{array}$ & $\begin{array}{l}\text { Coefficient de } \\
\text { transfert du }{ }^{137} \mathrm{Cs} \\
\left(\mathrm{Bq} \mathrm{kg} \mathrm{kg}^{-1} \text { frais }\right. \\
\left./ \mathrm{Bq}^{-2} \mathrm{~m}^{-2} \text { sol }\right)\end{array}$ \\
\hline \multicolumn{5}{|l|}{ Cèpes $n^{\circ} 1$} \\
\hline Sol Cèpes n ${ }^{\circ} 1$ : & $0-15 \mathrm{~cm}$ & 0,10 & 0,025 & $0,53 \times 10^{-3}$ \\
\hline \multicolumn{5}{|l|}{ Coulemelles } \\
\hline Sol Coulemelles : & $0-20 \mathrm{~cm}$ & 0,13 & 0,005 & $0,04 \times 10^{-3}$ \\
\hline Cèpes n ${ }^{\circ} 2$ & & non disponible & non disponible & non disponible \\
\hline Cèpes n³ & & 0,07 & 0,17 & $2,18 \times 10^{-3}$ \\
\hline Sol Cèpes n³: & $0-20 \mathrm{~cm}$ & & & \\
\hline Cèpes n ${ }^{\circ} 4$ & & 0,06 & 0,09 & $1,57 \times 10^{-3}$ \\
\hline Chanterelles & & 0,05 & 0,21 & $3,60 \times 10^{-3}$ \\
\hline Girolles $n^{\circ} 1$ & & 0,09 & 0,11 & $1,85 \times 10^{-3}$ \\
\hline Sol associé : & $0-10 \mathrm{~cm}$ & & & \\
\hline \multicolumn{5}{|l|}{ Girolles $n^{\circ} 2$} \\
\hline Sol Girolles $n^{\circ} 2$ : & $0-20 \mathrm{~cm}$ & 0,21 & 0,08 & $0,83 \times 10^{-3}$ \\
\hline \multicolumn{5}{|l|}{ Lactaires délicieux } \\
\hline Sol Lactaires déli & $0-20 \mathrm{~cm}$ & 0,09 & 0,46 & $1,42 \times 10^{-3}$ \\
\hline \multicolumn{5}{|l|}{ Myrtilles } \\
\hline Sol Myrtilles : & $0-20 \mathrm{~cm}$ & 0,02 & 0,01 & $0,06 \times 10^{-3}$ \\
\hline
\end{tabular}

TABLEAU V

Facteurs de transfert du ${ }^{40} \mathrm{~K}$ et du ${ }^{137} \mathrm{Cs}$ pour quelques champignons. Transfer factors of ${ }^{40} \mathrm{~K}$ and ${ }^{137} \mathrm{Cs}$ for some mushrooms.

\begin{tabular}{|l|c|c|}
\hline Espèces & Facteurs de transfert $\left(\mathrm{Bq} \mathrm{kg}^{-1}\right.$ frais / $\mathrm{Bq} \mathrm{kg}^{-1}$ sol sec $)$ \\
\hline Boletus edulis & ${ }^{40} \mathrm{~K}$ & ${ }^{137} \mathrm{Cs}$ \\
\hline Gomphidius glutinosus & 0,17 & 0,07 \\
Rozites caperata & 0,17 & 0.15 \\
Russula integra & 0,16 & 3,2 \\
Suillus luteus & 0,27 & 0,005 \\
Xerocomus badius & 0,2 & 1,87 \\
\hline
\end{tabular}

Le tableau IV regroupe les facteurs de transfert pour le ${ }^{137} \mathrm{Cs}$ et le ${ }^{40} \mathrm{~K}$ et le coefficient de transfert pour le ${ }^{137} \mathrm{Cs}$. 
Les facteurs de transfert pour le ${ }^{40} \mathrm{~K}$ sont relativement stables, avec un écart maximal d'environ un facteur 5, alors que les facteurs de transfert pour le ${ }^{137} \mathrm{Cs}$ sont extrêmement variables, d'un facteur d'environ 100. Ceci est en parfait accord avec les données de Heinrich (1992) qui a relevé les facteurs de transfert à la fois pour le potassium et le césium. Les résultats sont présentés dans le tableau V.

On peut s'apercevoir facilement que les facteurs de transfert pour le ${ }^{40} \mathrm{~K}$ fluctuent peu à l'intérieur de limites étroites alors que ceux du ${ }^{137} \mathrm{Cs}$ varient largement. Les mécanismes gouvernant l'accumulation du césium dans les champignons ne sont pas connus, mais Bakken et Olsen (1990) proposent l'hypothèse suivante : dans la membrane cellulaire du mycélium de certains champignons, les enzymes de transport présenteraient des propriétés de discrimination entre les ions $\mathrm{Cs}^{+}$et $\mathrm{K}^{+}$, ce qui pourrait expliquer les différences importantes entre espèces de champignons.

Une autre hypothèse pourrait être également l'existence dans certaines espèces de champignons de composés chimiques, comme des dérivés de l'acide pulvinique chez Xerocomus badius, qui présentent une très grande affinité pour le cesium (Heinrich, 1993).

En comparaison avec des coefficients de transfert obtenus dans diverses publications internationales, les valeurs trouvées dans le Boréon sont, globalement 5 à 130 fois inférieures à celles publiées (voir Tab. VI). Ce constat peut s'expliquer soit par l'importance des retombées des anciens tirs, dont on dit habituellement que le césium n'est pratiquement plus disponible, soit par une tendance à une diminution des coefficients de transfert en fonction du temps, soit encore à des caractéristiques physico-chimiques du sol qui rendent le cesium moins disponible.

TABLEAU VI

Coefficients de transfert obtenus dans le Massif du Boréon comparés à ceux de la littérature internationale.

Transfer coefficients obtained in the Boreon massif compared to international references.

\begin{tabular}{|l|c|c|}
\hline & \multicolumn{2}{|c|}{$\begin{array}{c}\text { Coefficients de trausfert }\left(\mathrm{m}^{2} \mathrm{~kg}^{+1} \mathrm{sec}\right) \\
\text { Valeurs publiées (1) }\end{array}$} \\
\hline Champignons et baies & Massif du Boréon & 0,09 \\
\hline Cantharellus cibarius & 0,02 (moyenne) & 0,2 \\
\hline Cantharellus lutescens & 0,02 (moyenne) & 0,5 \\
\hline Lactarius deliciosus & 0,07 & Lactarius sp. $: 0,5-9,0$ \\
\hline Macrolepiota procera & 0,02 & 0,006 \\
\hline Vaccinium myrtillus & 0,0003 & 0,08 \\
\hline
\end{tabular}

(1) : Bakken et Olsen, 1990 ; Battiston et al., 1989 ; Block et Pimpl, 1990 ; Byrne, 1988 ; Fawaris et Johanson, 1994 et 1995 ; Henrich et al., 1990 ; Horyna et Randa, 1988 ; Hove et al., 1990 ; IAEA, 1994 ; Kenigsberg et al., 1995 ; Lambinon et al., 1988 ; Mascanzoni, 1990 ; Randa et al., 1990; Rantavaara, 1990, Römmelt et al., 1990 ; Svadlenkova, 1996. 


\section{Conclusion}

Lors de cette mission d'étude, il a été possible de :

- disposer de valeurs de contamination de certaines espèces de champignons, de baies, en référence au sol sur lequel ils ont poussé, dans un endroit précis du Parc National du Mercantour;

- déterminer les rapports isotopiques, les facteurs et coefficients de transfert afin d'évaluer les dépôts, les phénomènes de migration dans les sols, les transferts aux champignons et aux baies et les comparer aux valeurs de la littérature internationale;

- montrer que les teneurs en césium, même si elles sont significatives, restent très inférieures aux concentrations maximales admissibles.

Remerciements : Cette campagne d'échantillonnage a été entreprise en collaboration avec le Parc National du Mercantour et grâce à l'aide du secteur de SaintMartin Vésubie. Le laboratoire d'études radioécologiques continentales et de la méditerranée (LERCM) de Cadarache a participé aux prélèvements sur le terrain et au conditionnement des échantillons. Les mesures de spectrométrie gamma ont été réalisées par le laboratoire de mesure de la radioactivité de l'environnement (LMRE) d'Orsay.

\section{RÉFÉRENCES}

Bakken L.R., Olsen R.A. (1990) Accumulation of Radiocaesium in fruit bodies of fungi. Dans : Transfer of radionuclides in natural and semi-natural environments (G. Desmet, P. Nassimbeni, M. Belli, Eds) pp. 664-668. Elsevier, Londres.

Battiston G.A., Degetto S., Gerbasi R., Sbrignadello G. (1989) Radioactivity in mushrooms in Northeast Italy following the Chernobyl accident. J. Environ. Radioact. 9, 53-60.

Block J., Pimpl M. (1990) Cycling of radiocesium in two forest ecosystems in the state of RhinelandPalatinate. Dans: Transfer of radionuclides in natural and semi-natural environments $(\mathrm{G}$. Desmet, P. Nassimbeni, M. Belli, Eds) pp. 450-458. Elsevier, Londres.

Byrne A.R. (1988) Radioactivity in fungi in Slovenia, Yugoslavia, following the Chernobyl accident. J. Environ. Radioact. 6, 177-183.

Fawaris B.H., Johanson K.J. (1994) Radiocaesium in soil and plants in a forest in central Sweden. Sci. Total Environ. 157, 133-138.

Fawaris B.H., Johanson K.J. (1995) A comparative study on Radiocaesium $\left({ }^{137} \mathrm{Cs}\right)$ uptake from coniferous forest soil. J. Environ. Radioact. 28, 313-326.

Heinrich G. (1992) Uptake and transfer factors of ${ }^{137}$ Cs by mushrooms. Radiat. Environm. Biophys. 31, 39-49.

Heinrich G. (1993) Distribution of radiocesium in the different parts of mushrooms. J. Environ. Radioactivity, 18, 229-245.

Henrich E., Friedrich M., Haider W., Kienzl K., Hiesel E., Boisits A., Hekerle G. (1990) The contamination of large Austrian forest systems after the Chernobyl nuclear reactor accident: studies 1988 and further. In: Transfer of radionuclides in natural and semi-natural environments (G. Desmet, P. Nassimbeni, M. Belli, Eds) pp. 217-225. Elsevier, Londres. 


\section{Ph. CALMON}

Horyna J., Randa Z. (1988) Uptake of radiocesium and alkali metals by mushrooms. J. Radioanal. Nucl. Chem. Lett. 127 (2), 107-120.

Hove K., Pedersen O., Garmo T.H., Hansen H.S., Staaland H. (1990) Fungi : a major source of radiocesium contamination of grazing ruminants in Norway. Health Physics 59, 189-192.

AIEA (1994) Handbook of parameter values for the prediction of the radionuclide transfer in temperate environments, technical report series $\mathrm{N}^{\circ} 364$. AIEA, Vienna.

Kenigsberg Y.E., Buglova E.E., Shevchuk V.E., Renaud Ph., Maubert H. (1995) Activity in foodstuffs from natural environments and activity changes due to culinary practices. In: Joint Study Project 5 (JSP-5). Pathway Analysis and Dose Distributions (Final report for the contracts COSU-CT930053 and COSU-CT94-0091 of the European Commission) pp. 63-86.

Lambinon J., Fraiture A., Gasia M.C., Guillitte O. (1988) La radiocontamination des champignons sauvages en Wallonie (Belgique) suite à l'accident de Tchernobyl. Dans : Impact des accidents d'origine nucléaire sur l'environnement, 14-18 mars 1988, Cadarache, tome 2. (Centre d'études nucléaires de Cadarache) pp. E 37-44.

Mascanzoni D. (1990) Uptake of ${ }^{90} \mathrm{Sr}$ and ${ }^{137} \mathrm{Cs}$ by mushrooms following the Chernobyl accident. Dans : Transfer of radionuclides in natural and semi-natural environments (G. Desmet, P. Nassimbeni, M. Belli, Eds) pp. 459-467. Elsevier, Londres.

Randa Z., Benada J., Horyna J., Klan J. (1990) Mushrooms : significant source of internal contamination by radiocaesium. In: Transfer of radionuclides in natural and semi-natural environments (G. Desmet, P. Nassimbeni, M. Belli, Eds) pp. 169-178. Elsevier, Londres.

Rantavaara A.H. (1990) Transfer of radiocaesium through natural ecosystems to foodstuffs of terrestrial origin in Finland. In: Transfer of radionuclides in natural and semi-natural environments (G. Desmet, P. Nassimbeni, M. Belli, Eds) pp. 202-209. Elsevier, Londres.

Römmelt R., Hiersche L., Schaller G., Wirth E. (1990) Influence of soil fungi (basidiomycetes) on the migration of $\mathrm{Cs}^{134+137}$ and $\mathrm{Sr}^{90}$ in coniferous forest soils. In: Transfer of radionuclides in natural and semi-natural environments (G. Desmet, P. Nassimbeni, M. Belli, Eds) pp. 152-160. Elsevier, Londres.

Svadlenkova M., Konecny J., Smutny V. (1996) Model calculation of radiocaesium transfer into food products in semi-natural forest ecosystems in the Czech Republic after a nuclear reactor accident and an estimate of the population dose burden. Environmental Pollution 92, 173-184. 